\title{
Fas determines outcome in DLBCL
}

Diffuse-large B-cell lymphoma (DLBCL) is a particularly aggressive form of non-Hodgkin lymphoma. CHOP chemotherapy and rituximab have increased the response rates and survival outcomes in elderly patients with DLBCL. Many investigators have tried to identify prognostic factors for such patients to distinguish those with a favorable or unfavorable outcome and to select appropriate treatment strategies.

\section{4 ....serum soluble Fas levels} is a significant prognostic factor and could be a useful tool... 77

Before the introduction of rituximab into clinical practice, Hara et al. reported that serum soluble Fas levels were prognostic. Now, the same group has shown that soluble Fas levels are prognostic in patients who receive rituximab-based $\mathrm{CHOP}$ chemotherapy. In total, 132 patients received $\mathrm{CHOP}$ chemotherapy without rituximab (group A) and 75 received rituximab (group B). In group A, the complete response rates were $82 \%$ and $51 \%$ for patients with soluble Fas $<3.0$ and $\geq 3.0 \mathrm{ng} / \mathrm{ml}$. The complete response rates did not differ significantly in patients with high or low soluble Fas levels in group B. The respective 5-year overall survival for patients with $<3.0$ and $\geq 3.0 \mathrm{ng} / \mathrm{ml}$ in group A was $66 \%$ and $33 \%$. The 3 -year overall survival for patients with $<3.0$ and $\geq 3.0 \mathrm{ng} / \mathrm{ml}$ in group B was $79 \%$ and $45 \%$.

The researchers conclude that serum soluble Fas levels are a significant prognostic factor and could be a useful tool in the selection of appropriate treatment strategies.

\section{Lisa Hutchinson}

Original article Hara, T. et al. Serum soluble Fas leve determines clinical outcome of patients with diffuse large B-cell lymphoma treated with CHOP and R-CHOP. J. Cancer Res. Clin. Oncol. 135, 1421-1428 (2009). 